ARTICLE

\title{
Photobase effect for just-in-time delivery in photocatalytic hydrogen generation
}

\author{
Jiawen Fang (1) ${ }^{1}$, Tushar Debnath (10) ${ }^{1}$, Santanu Bhattacharyya (i) ${ }^{1,3}$, Markus Döblinger ${ }^{2}$, Jochen Feldmann ${ }^{1} \&$
} Jacek K. Stolarczyk (iD) ${ }^{1 \times}$

Carbon dots (CDs) are a promising nanomaterial for photocatalytic applications. However, the mechanism of the photocatalytic processes remains the subject of a debate due to the complex internal structure of the CDs, comprising crystalline and molecular units embedded in an amorphous matrix, rendering the analysis of the charge and energy transfer pathways between the constituent parts very challenging. Here we propose that the photobasic effect, that is the abstraction of a proton from water upon excitation by light, facilitates the photoexcited electron transfer to the proton. We show that the controlled inclusion in CDs of a model photobase, acridine, resembling the molecular moieties found in photocatalytically active CDs, strongly increases hydrogen generation. Ultrafast spectroscopy measurements reveal proton transfer within 30 ps of the excitation. This way, we use a model system to show that the photobasic effect may be contributing to the photocatalytic $\mathrm{H}_{2}$ generation of carbon nanomaterials and suggest that it may be tuned to achieve further improvements. The study demonstrates the critical role of the understanding the dynamics of the CDs in the design of next generation photocatalysts.

\footnotetext{
${ }^{1}$ Chair for Photonics and Optoelectronics, Nano-Institute Munich, Department of Physics, Ludwig-Maximilians-Universität (LMU), Königinstr. 10, 80539 Munich, Germany. ${ }^{2}$ Department of Chemistry, Ludwig-Maximilians-Universität München, Butenandtstr. 5-13 (E), 81377 Munich, Germany. ${ }^{3}$ Present address: Department of Chemical Sciences, IISER Berhampur, Transit Campus (Govt. ITI Building), Engg. School Junction, Berhampur, Odisha 760010, India.

凶email: jacek.stolarczyk@physik.uni-muenchen.de
} 
$\mathrm{n}$ photocatalytic hydrogen generation the energy of an incident photon is utilized to drive the energetically up-hill water splitting reaction ${ }^{1,2}$. The evolved hydrogen can then serve directly as an energy-rich fuel or as a reactant in chemical synthesis of carbon-based fuels, e.g. methane in the Sabatier or Fischer-Tropsch processes ${ }^{3,4}$. This approach is an appealing alternative to harvest and store abundant solar energy, potentially providing a renewable solution for the global energy supply ${ }^{5}$. In general, the photocatalytic reaction involves a complex sequence of charge carrier (electron and hole) generation, separation and transfer steps, and typically requires a concurrent proton transfer. The efficiency of such proton-coupled electron transfer (PCET) processes is limited by its slowest component, which could be reactant diffusion ${ }^{6}$. Consequently, a major challenge in photocatalytic $\mathrm{H}_{2}$ production is to ensure that all participants in the process (photoexcited charge carriers, protons, and possibly other molecules) are delivered to the reaction site at optimal time. Otherwise, the photoexcited carriers may recombine, photodegrade the catalysts or induce unwanted side reactions, in all cases reducing the activity.

Photobases are molecules that have higher proton affinity in the excited state than in the ground state. In other words, the $\mathrm{pK}_{\mathrm{a}}$ in the excited state is much higher than in the ground state, so that upon photoexcitation the now stronger base abstracts a proton from the environment. Hence, such molecules offer an opportunity to control the proton transfer by light ${ }^{7,8}$. For instance, quinoline photobases have been shown to form protonated species within tens of picoseconds of the light excitation ${ }^{9}$. Effectively, the photobase can ensure that the proton promptly and selectively arrives at the reduction site. This result can also be achieved by other means, for example by lowering the $\mathrm{pH}$. Nonetheless, utilizing the excited state acid-base equilibria the same effect can conceivably be realized in a neutral medium, without the potentially corrosive acidic conditions. Therefore, for photocatalytic $\mathrm{H}_{2}$ generation the photobases present an appealing approach, wherein the incident photons not only provide the energy for the water splitting, but also dynamically alter the photocatalyst to enhance the process efficiency ${ }^{10}$.

Nanostructured photocatalysts, either inorganic, organic, or hybrid, rose to the forefront of the photocatalytic field because their composition, structure, and surface can be controlled to tailor the optoelectronic and morphological properties to the desired function ${ }^{11-13}$. Carbon dots (CDs) are a recent entrant into photocatalysis that has attracted attention due to their versatility, photostability, absence of heavy metals, ease of preparation, and tunable properties ${ }^{14,15}$. This flexibility derives from their complex internal structure, inherent disorder, and surface functionality. Effectively, small changes in the preparative procedure can result in large differences in the properties. While the application possibilities are impressive, there is still no detailed understanding of their structure and the mechanism of photocatalytic reactions ${ }^{16-18}$. They are generally considered to consist of aromatic, $s p^{2}$-hybridized domains immersed in a $s p^{3}$-hybridized amorphous matrix with various functional groups on the surface ${ }^{19,20}$. In this context, we have shown that the optical properties of CDs prepared from citric acid and ethylenediamine can be reproduced by a simple model of polycyclic hydrocarbons embedded in an amorphous polymer, poly(methyl methacrylate $)^{20}$. Heterocyclic compounds, including strong molecular fluorophores, form in the presence of nitrogen-containing precursors ${ }^{21,22}$. Importantly, the position of the nitrogen atom dopant in the heterocyclic aromatic structure, controlled by the synthetic procedure, determines the functionality of the CDs. Whilst the graphitic nitrogen increases the photoluminescence (PL) quantum yield, pyridinic and pyrrolic nitrogen yields much higher photocatalytic $\mathrm{H}_{2}$ generation activity ${ }^{23}$. Pyridine and its larger analogs, such as quinoline or acridine, are weak bases that can be protonated at the nitrogen atom in the excited state, suggesting that such photocatalytically active CDs contain photobasic moieties in their structure. Interestingly, heptazine (tri-striazine), the building block of graphitic carbon nitrides (g-CN), also contains multiple $\mathrm{N}$ atoms at the edge sites of the aromatic structure and exhibits photobasic behavior ${ }^{24,25}$. Recent calculations show that in the excited state heptazine molecules induce proton and electron transfer from hydrogen-bonded water molecules leading to heptazinyl radicals ${ }^{26,27}$. These species can undergo further photolysis, recovering the heptazine and releasing hydrogen radicals that further react to form molecular $\mathrm{H}_{2}$. In corroborating evidence, Electron Paramagnetic Resonance (EPR) experiments show that a long-lived radical forms in cyanamide-functionalized heptazine-based g-CN upon illumination that can be later, under dark conditions, used to produce $\mathrm{H}_{2}{ }^{28-30}$. It has been observed that protonation of the g-CN structure in acidic media increases the $\mathrm{H}_{2}$ evolution rate ${ }^{31-33}$. This further supports the argument that the proton transfer rate may be a limiting factor in the photocatalytic $\mathrm{H}_{2}$ generation on g-CN. Overall, these experimental and computational results offer tantalizing hints that the photobasic effect of the constituent units in CDs and in $\mathrm{g}$ $\mathrm{CN}$ is highly beneficial for the photocatalytic activity of these nanomaterials and at least partially responsible for their impressive $\mathrm{H}_{2}$ formation rates.

In this paper, we show that the introduction of photobasic units into carbon dots indeed increases the $\mathrm{H}_{2}$ generation rate, on the basis of time-resolved spectroscopy and photocatalytic activity measurements. Due to complexity of nitrogen-containing CDs, it is difficult to find an appropriate blank reference sample which exhibits no nitrogen-related acid-base activity. Therefore, we started with polyethylene glycol (PEG) derived nitrogen-free CDs and introduced acridine, a model photobase, into the photocatalyst. Acridine is an $N$-heterocyclic aromatic compound, resembling molecules likely present in the $\mathrm{CDs}^{20}$, with $\mathrm{pK}_{\mathrm{a}}=5.5$ in the ground state and $\mathrm{pK}_{\mathrm{a}}{ }^{*}=10.7$ in the excited state ${ }^{34-36}$. This means that at $\mathrm{pH} 7$ it is protonated to a very small extent (3\%), but becomes mostly protonated in the excited state (see details in the Supplementary Note 1). Calculations suggest that excitation of acridine in water induces proton-coupled electron transfer leading to acridinyl radical that can split under illumination to release a hydrogen radical ${ }^{37}$. A common acridine-based derivative photobase, acridine orange, can abstract a proton from alcohols coupled with an electron transfer that can also result in acridinylbased radicals ${ }^{38,39}$. This further implied that acridine in its excited protonated state is prone to further reduction reactions (e.g. accepting an electron), making it suitable for studying $\mathrm{H}_{2}$ generation from water ${ }^{10}$. These studies were focused on molecular interactions of acridine with a potential proton and electron donor (alcohol and water). In our work where acridine is integrated with CDs the process can be even more efficient. This is because the electron can also be readily transferred from the photoexcited CDs, which have a broader absorption range.

In short, we synthesize CDs which comprise a model photobase and we show that the photobasic effect can be a plausible element of the mechanism of photocatalytic hydrogen generation with CDs. The understanding of the mechanism demonstrates that careful design of the CDs enables control by light of the proton transfer rates at the photocatalyst surface to enhance the efficiency of $\mathrm{H}_{2}$ production. In this way, we believe it opens a new avenue for modifying the functionality of CDs to bring further improvements in efficiency.

\section{Results}

Preparation and characterization of CDs. The nitrogen-free CDs were prepared in the first step and then combined with a model photobase, acridine (cf. Fig. 1), to demonstrate an increase 
in photocatalytic activity. The CDs were synthesized in an autoclave from PEG dissolved in ethanol by pyrolysis at $200^{\circ} \mathrm{C}^{40}$. The details of the preparation are provided in the Methods sections. Following the synthesis, ethanol was evaporated and the CDs were dispersed in water. Transmission electron microscopy (TEM, see Fig. 2a and Supplementary Fig. 1) and dynamic light scattering (Supplementary Fig. 2) measurements reveal the formation of ultrasmall monodisperse particles $2 \mathrm{~nm}$ in diameter (inset in Fig. 2a). Upon close inspection, most particles exhibit crystal fringes, with lattice plane distances $\sim 2.35 \AA$, indicating an at least partially crystalline structure (Fig. 2b) ${ }^{23,41}$.

In the next step, the optical properties of the CDs were investigated. As the acid-base equilibrium is determined by the $\mathrm{pH}$ of the medium, we varied the proton concentration. We focused on three values of $\mathrm{pH}: 3,7$, and 13, chosen to represent the three relevant populations of acridine: always protonated $(\mathrm{pH}$ 3 , i.e. acridinium cation, $\mathrm{Acr}-\mathrm{H}^{+}$), mostly non-protonated in the ground state, but protonated to a larger extent in the excited state $(\mathrm{pH} 7)$, and always non-protonated $(\mathrm{pH} 13)$, consistently with the excited state populations reported from fluorescence timeresolved studies ${ }^{42}$. In effect, these $\mathrm{pH}$ values are on either side of acridine $\mathrm{pK}_{\mathrm{a}}$ and $\mathrm{pK}_{\mathrm{a}}{ }^{*}$ (5.5 and 10.7, respectively). For

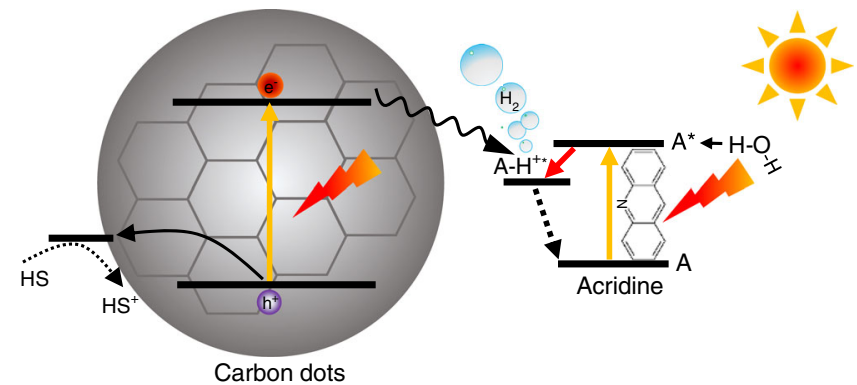

Fig. 1 Photobase effect. Schematic of the $\mathrm{H}_{2}$ generation on the CD-acridine photocatalyst in which, upon light excitation, acridine (A) abstracts a proton from water. reference purposes, the same values were used also for the nitrogen-free CDs. The absorption spectra of the CDs, presented in Fig. 2c, do not exhibit any significant dependence on $\mathrm{pH}$. They are nearly featureless, but contain a long absorption tail extending weakly into the visible part of the spectrum. The CDs are photoluminescent at all $\mathrm{pH}$ values, with a single $\mathrm{PL}$ peak in every spectrum. However, the Stokes shift is $\mathrm{pH}$-dependent, with the PL maxima at between $344 \mathrm{~nm}(\mathrm{pH} \mathrm{13})$ and $375 \mathrm{~nm}(\mathrm{pH} \mathrm{3})$. The dependence of PL on proton concentration is in agreement with literature and can be attributed to the presence of hydroxyl groups on the surface of the $\mathrm{CDs}^{43}$. The Fig. $2 \mathrm{~d}$ shows the absorption and PL spectra of the second component of the photocatalyst, i.e. of acridine. The sharp absorption peaks at 354 $\mathrm{nm}$ can be ascribed to transitions to singlet state $\mathrm{S}_{2}$, whose energy is largely unaffected by the changes in $\mathrm{pH}^{35,44}$. This absorption range is very convenient for the excitation during the optical spectroscopy experiments. In contrast to $S_{2}$, the energy level of $S_{1}$ state is lower in the protonated state, leading to a pronounced red-shift at $\mathrm{pH} 3$ of the secondary peak at longer wavelengths ${ }^{35}$. As mentioned before, acridine is mostly non-protonated at $\mathrm{pH} 7$. Hence, the normalized absorption spectrum in neutral conditions is identical to the spectrum acquired at $\mathrm{pH} \mathrm{13,} \mathrm{with} \mathrm{the} \mathrm{exception}$ of a small peak at $430 \mathrm{~nm}$ due to a small fraction of the protonated species. The PL quantum yield of acridine is strongly dependent on $\mathrm{pH}$, with the yield decreasing with $\mathrm{pH}^{35,45}$. The spectra are also different with the peak of emission of the protonated species $(\mathrm{pH} \mathrm{3})$ at $475 \mathrm{~nm}$ and of the non-protonated species $(\mathrm{pH} \mathrm{13})$ at $427 \mathrm{~nm}$. The photobasic behavior of acridine manifests itself in the $\mathrm{PL}$ of the sample at $\mathrm{pH} 7$, which comprises the emission peaks of both species. In comparison to absorption, where the spectra of samples at $\mathrm{pH} 7$ and $\mathrm{pH} 13$ are nearly identical, PL emission at $\mathrm{pH} 7$ appears red-shifted. The nonnormalized emission spectra (see Supplementary Fig. 3) prove that, despite the higher PL quantum yield of protonated acridine, the small fraction (3\%) of the protonated species at $\mathrm{pH} 7$ cannot account for the shift alone. Hence, it is clear that a substantial part of acridine molecules become protonated in the excited state before the radiative charge recombination. This is confirmed by
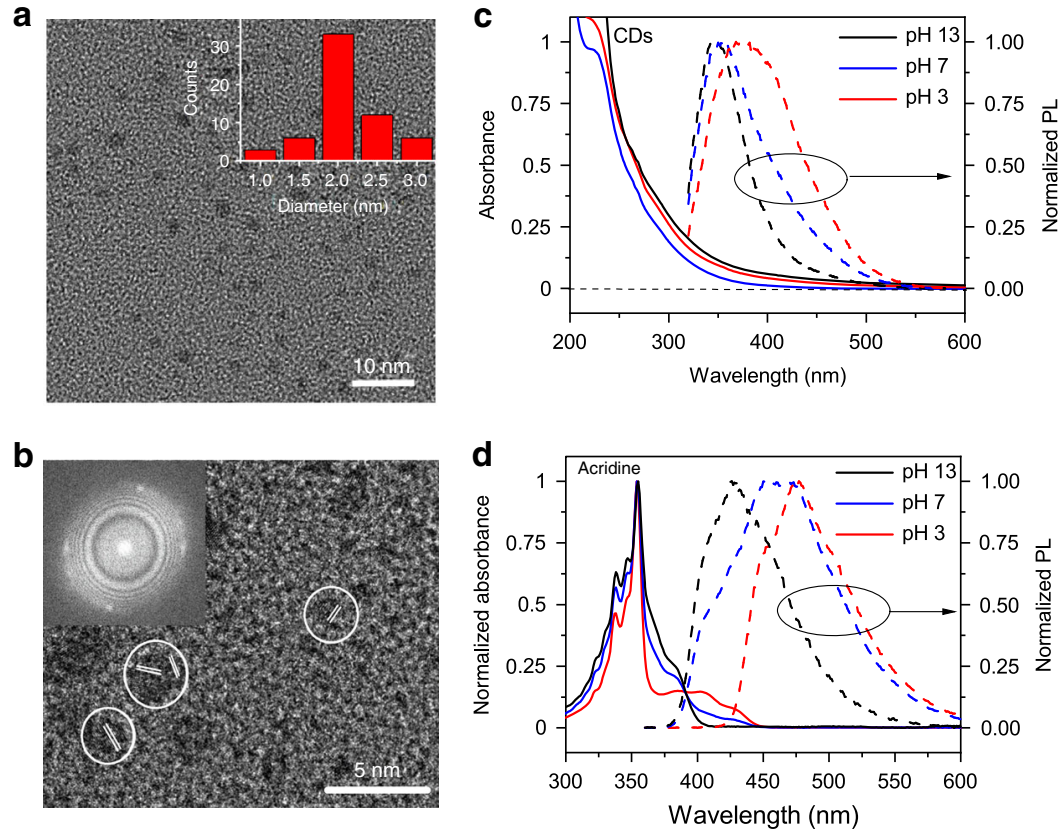

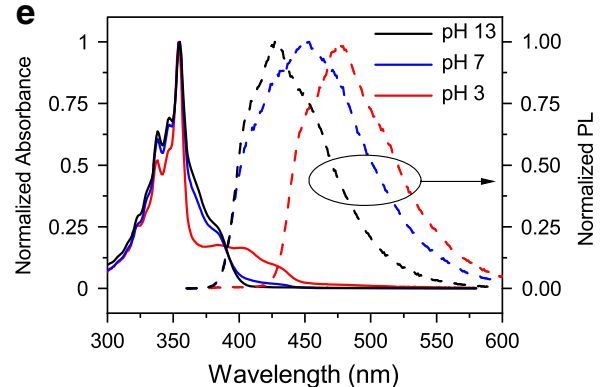

$\mathbf{f}$

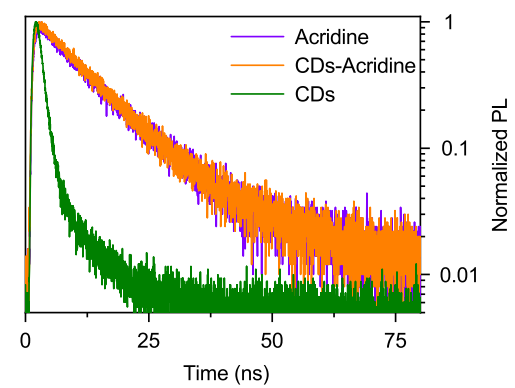

Fig. 2 Morphological and optical properties of CDs. a TEM image of CDs (size distribution in inset); $\mathbf{b}$ HR-TEM image of CDs (FFT image in inset). UV-Vis absorption (solid lines) and photoluminescence (dashed lines) spectra of $\mathbf{c} C D$ s, $\mathbf{d}$ acridine, and $\mathbf{e} \mathrm{CD}$-acridine at different $\mathrm{pH}$ values. $\mathbf{f}$ Time-resolved $\mathrm{PL}$ decay of $C D s$, acridine, and $C D$-acridine, measured at $450 \mathrm{~nm}$. For the acquisition of $\mathrm{PL}$, in all cases the samples were excited at $350 \mathrm{~nm}$. 
measurements of weakened PL emission of acridine in media of higher $\mathrm{pK}_{\mathrm{a}}$ (ethanol, 15.6) and increased intensity in media of lower $\mathrm{pK}_{\mathrm{a}}\left(\mathrm{NH}_{4} \mathrm{Cl}\right.$ solution, $\mathrm{pK}_{\mathrm{a}}=9.2$ and boric acid solution, $\mathrm{pK}_{\mathrm{a}}=9.2$ ), shown in Supplementary Fig. 4.

The combined CD-acridine samples were prepared in two ways. In one method, the CDs were synthesized first, transferred to water, and mixed with an aqueous solution of acridine. This approach was designed to adsorb acridine on the surface of the CDs. In the second method, acridine (same amount as above) was mixed with the precursor of the CDs, PEG, and the synthetic procedure was carried out as before. After the carbonization, acridine could be located within the CDs either as free molecules or incorporated into larger $\mathrm{N}$-hetorocyclic domains, mimicking the proposed composition and structure of $\mathrm{N}$-doped CDs prepared from citric acid and diamines ${ }^{20}$. Due to different modes of acridine inclusion in this method, complicating the understanding of individual contributions, we used the second method as reference samples only, focusing in the further analysis on the first method that leads to CDs with acridine adsorbed on the outside. The absorption and PL spectra of this CD-acridine sample (see Fig. 2e) show strong resemblance to the free acridine sample. The comparison of non-normalized absorption spectra of CD-acridine with its individual components (see Supplementary Fig. 5) confirms a dominant contribution of acridine in the range 325-400 nm, but a significant contribution of CDs for shorter and longer wavelengths where acridine does not absorb. To investigate the role of the PL emission from the CDs in this combined system, we acquired the PL excitation (PLE) spectrum at $355 \mathrm{~nm}$ (see Supplementary Fig. 6). This wavelength was chosen to match the free CDs emission (cf. Fig. 2c). Indeed, the PLE spectrum shows the emission originating at $286 \mathrm{~nm}$, corresponding to the absorption by the aromatic structures in the CDs. Interestingly, in the combined sample, the PL at $350 \mathrm{~nm}$ is quenched (cf. Supplementary Fig. 7), suggesting energy or electron transfer from CDs to acridine. The Forster energy transfer pathway appears possible because the $350 \mathrm{~nm}$ is very close to the peak acridine absorption. Hence, in a further experiment, we measured the PLE spectra setting the detection wavelength to $450 \mathrm{~nm}$, i.e., at peak acridine emission. As shown in Supplementary Fig. 8, the PLE of the CD-acridine system is higher than of acridine alone in the region $280-320 \mathrm{~nm}$ that coincides with strong $\mathrm{CD}$ absorption. The emission of CDs at $450 \mathrm{~nm}$ is weak, implying that indeed the energy transfer from $\mathrm{CDs}$ to acridine can take place. Although the time-correlated single photon counting (TCSPC) measurements at $450 \mathrm{~nm}$ (Fig. 2f) show that the PL decay dynamics of the CD-acridine sample is the same as in free acridine, this does not contradict the electron transfer, because the native $\mathrm{CD}$ lifetime is shorter than of acridine. To verify the possibility of electron transfer, we performed cyclic voltammetry measurements of CDs and acridine in mildly alkaline conditions to determine the relative positions of the conduction band edge of the CDs versus the LUMO level of acridine. In the voltammograms these levels are manifested as cathodic (reduction) peaks. Supplementary Figure 9 reveals that there are prominent cathodic peaks at around $-1.03 \mathrm{~V}$ and $-0.92 \mathrm{~V}$ vs $\mathrm{Ag} / \mathrm{AgCl}$ for $\mathrm{CDs}$ and acridine, respectively. Assuming that no significant reorganization occurs in the excited state of CDs, the reduction potential in the ground state gives a good representation of the oxidation potential in the excited state. Hence, the voltammetry results imply $\sim 100 \mathrm{meV}$ difference in the energy levels that enables electron transfer from excited CDs to the LUMO of non-protonated acridine. Importantly, this difference is even larger in the acidic conditions because the LUMO level of protonated acridine is lower, providing a stronger driving force ${ }^{36,46}$. Overall, these measurement suggest that both energy and electron transfers can take place. The second important conclusion to be drawn from the PL measurements of the combined system is that the photobasic properties of acridine should be similar in both free acridine and in the CDacridine sample. This means that free acridine can be treated as a good starting point for the investigation of the charge carrier and proton transfer dynamics.

Proton transfer rate. Transient absorption spectroscopy was then used to determine the rate of proton transfer in the excited state of acridine-based systems. To this end, the acridine solutions in water at different $\mathrm{pH}$ values were excited with $100 \mathrm{fs}$ pump pulses at $350 \mathrm{~nm}$ and the differential absorption spectra were acquired with a broad band pulse after a time delay up to 2.0 ns (see Fig. 3 ). The spectrum at $\mathrm{pH} 3$ contains two excited state absorption (ESA) maxima, at $455 \mathrm{~nm}$ and $512 \mathrm{~nm}$, respectively. The intersystem crossing rate is considered to be very low in the acridinium cation ${ }^{9}$, therefore the peaks are attributed to excitations from $S_{1}$ to $S_{n}$ for the protonated and non-protonated species, respectively 47 . The singlet spin multiplicity of the molecules is corroborated by the presence of a negative $\triangle \mathrm{OD}$ region at $530-570 \mathrm{~nm}$. There is no absorption of the protonated species in this region, but it overlaps with the low-energy tail of the PL emission. Therefore, it can be ascribed to stimulated emission of the singlet $\mathrm{Acr}-\mathrm{H}^{+}$. The presence of the minor ESA peak at 512 $\mathrm{nm}$ indicates a presence of a small fraction of non-protonated acridine even at $\mathrm{pH}$ 3. This assignment can be inferred from the position of the corresponding peak observed at $518 \mathrm{~nm}$ at $\mathrm{pH} 7$ and 13 (Fig. 3c, e), with the shift caused by the overlap with the strong emission of Acr-H+ in acidic conditions. To ascertain the claim, we performed an analogous experiment at lower $\mathrm{pH}$, where the non-protonated acridine would not be expected at all. Indeed, as shown in Supplementary Fig. 10, the peak vanishes completely at $\mathrm{pH} 1$ and the broad stimulated emission has an onset already at $520 \mathrm{~nm}$, confirming the assignment. Meanwhile, at alkaline conditions (Fig. 3e), the PL is much weaker and no stimulated emission regions can be seen. This is in agreement with a faster intersystem crossing and low PL quantum yield of the $S_{1}$ state expected in the non-protonated species $^{35,36,44}$. The resulting higher triplet state then relaxes to lower lying $\mathrm{T}_{1}$ state $^{36}$. The two ESA peaks at $518 \mathrm{~nm}$ and $440 \mathrm{~nm}$ are then attributed to the excitation from the $S_{1}$ to $S_{n}$ and from $T_{1}$ to $T_{n}$, respectively (see Supplementary Fig. 11) ${ }^{47}$. No traces of protonation are observed, as expected at this $\mathrm{pH}$. This was also confirmed by taking the measurements in ethanol which is harder to deprotonate than water (see Supplementary Fig. 12). The differential spectrum in ethanol contains the same ESA peaks with a very clear conversion between then, consistent with a transition from $S_{1}$ to $T_{1}$. The same process, albeit on a smaller scale, is also visible in water. The differential spectra at $\mathrm{pH} 7$ (see Fig. 3c) are broadly similar to the ones acquired at $\mathrm{pH} \mathrm{13,} \mathrm{which} \mathrm{is} \mathrm{in} \mathrm{agreement} \mathrm{with} \mathrm{the} \mathrm{mostly}$ deprotonated state of acridine at these conditions.

To inspect the dynamics of system more closely, the transient absorption traces of the acridine were plotted in Fig. 3 for several relevant wavelengths. The aim was to detect the formation of the protonated species, expected $\sim 450 \mathrm{~nm}$ (cf. Fig. 3a). However, at $440 \mathrm{~nm}$ the traces are essentially the same at $\mathrm{pH} 7$ and $\mathrm{pH} 13$, in contrast to $\mathrm{pH} 3$. This is because the signal is then dominated by the rise, after $\sim 65 \mathrm{ps}$, of the ESA peak corresponding to $\mathrm{T}_{1}$ to $\mathrm{T}_{\mathrm{n}}$ transition of non-protonated acridine. This rise is absent in acidic conditions. To shift away from this ESA peak, in Fig. 3d we plotted the traces recorded at $465 \mathrm{~nm}$. Here it becomes clear, that the signal at $\mathrm{pH} 7$ follows the dynamics of the non-protonated acridine until $30 \mathrm{ps}$ (cf. Supplementary Table 2), when the two traces start to diverge. There is no rise of the $T_{1}$ to $T_{n}$ peak, suggesting that acridine became protonated. From this time, the 

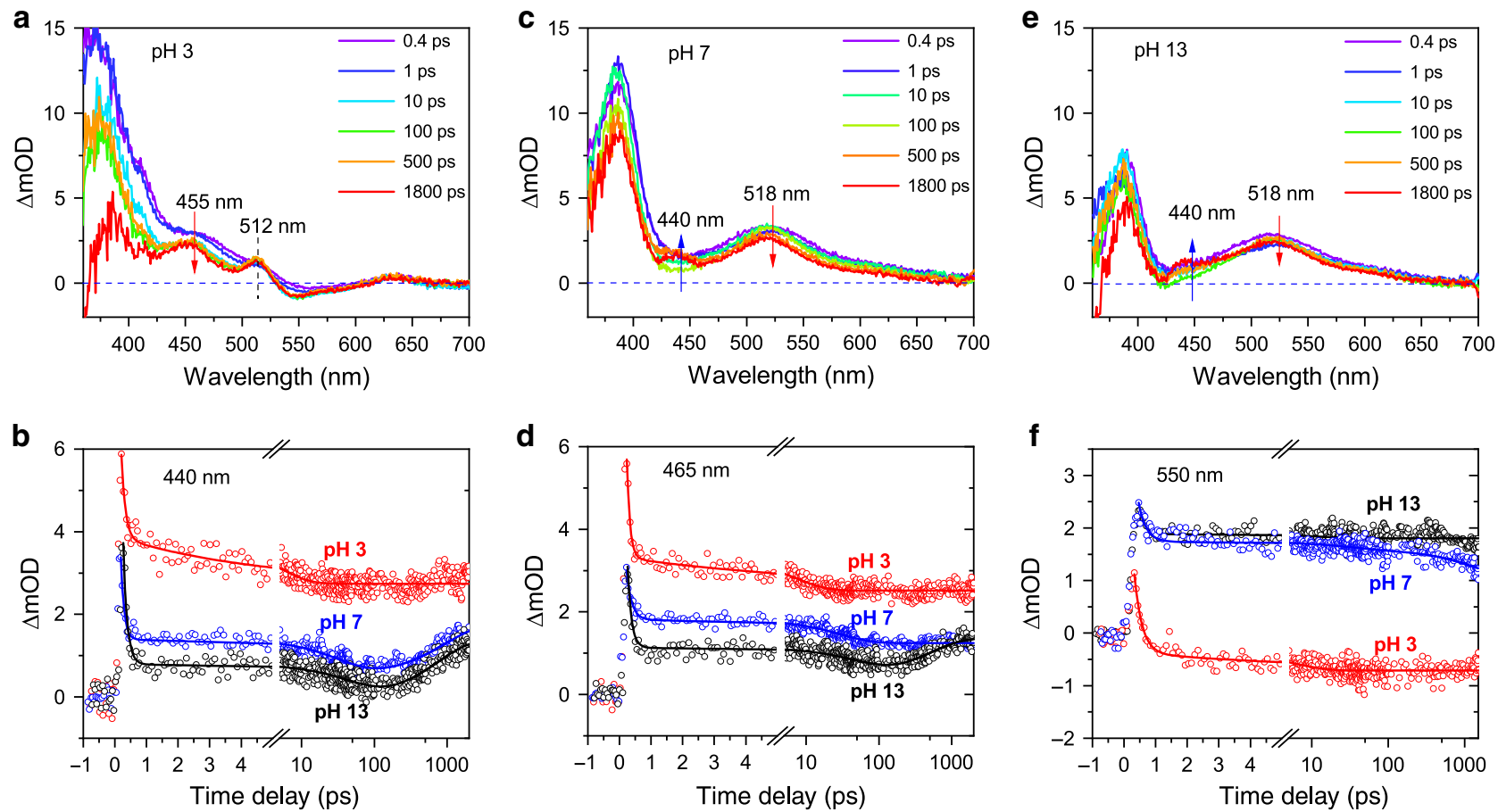

Fig. 3 Protonation dynamics. Transient absorption spectra of acridine at a pH 3, c pH 7, and e pH 13. Comparison of the transient absorption traces at b $440 \mathrm{~nm}$, d $465 \mathrm{~nm}$, and $\mathbf{f} 550 \mathrm{~nm}$ at pH 3, pH 7, and pH 13. Open circles represent the data points, the numerical fits are plotted as the solid lines.

dynamics follows that of the $\mathrm{pH} 3$ sample. The time scale of $30 \mathrm{ps}$ is in remarkable agreement with the protonation time scale reported earlier for quinolone (28 ps), a lower molecular mass analog of acridine $e^{7,9}$. A similar time scale can be extracted from the dynamics monitored at $550 \mathrm{~nm}$ (Fig. 3f), where the stimulated emission is observed at $\mathrm{pH} 3$ in the protonated sample, seen as a nearly constant negative $\Delta \mathrm{OD}$ signal. At $\mathrm{pH} 7$ the signal is positive with dynamics initially similar to $\mathrm{pH} 13$. However, an additional decay component arises $\sim 30$ ps resulting in a faster decrease of the $\Delta \mathrm{OD}$ signal. Such change is consistent with the protonation of acridine and the ensuing increase in PL emission by the acridinium cation. Importantly, while the analysis of the transient absorption measurements of the $\mathrm{CD}$-acridine sample is complicated by the contributions of the CDs themselves, a very similar protonation rate emerges from the transient trace plotted at $465 \mathrm{~nm}$ (see Supplementary Fig. 13). Therefore, the spectroscopic analysis provides evidence that the protonation time of acridine (free or adsorbed at the surface of CDs) at $\mathrm{pH} 7$ is only $\sim 30$ ps. Remarkably, this time is comparable with electron transfer to co-catalyst in many inorganic semiconductor-based photocatalysts and faster than photogenerated hole transfer in such systems ${ }^{48,49}$. Consequently, owing to the photobasic effect, the proton transfer could no longer be a rate-limiting step in photocatalytic $\mathrm{H}_{2}$ generation.

Photocatalytic $\mathbf{H}_{2}$ evolution. In order to verify that the inclusion of a photobasic moiety affects the $\mathrm{H}_{2}$ generation rate, we illuminated the samples with a Xenon lamp and measured the evolved hydrogen (see Methods section for details). Methanol was used as a hole scavenger. As shown in Fig. 4, the rate of $\mathrm{H}_{2}$ generation at $\mathrm{pH} 7$ is indeed much faster for the combined $\mathrm{CD}$-acridine sample $\left(0.032 \mu \mathrm{mol} \mathrm{h}^{-1}\right)$ than for CDs only $\left(0.08 \mu \mathrm{mol} \mathrm{h}^{-1}\right)$ or acridine itself $\left(0.08 \mu \mathrm{mol} \mathrm{h}^{-1}\right)$. The increase in the rate is also obvious when the amount of $\mathrm{H}_{2}$ is normalized by the mass of either the $\mathrm{CDs}$ or of acridine (Supplementary Fig. 14). The increase is observed irrespective of the choice of the preparation method of the CDacridine. In fact, it is even stronger (10-fold) for the sample where acridine is partially embedded inside the CDs (Supplementary Fig. 15). This implies that the stronger interaction between CDs and acridine, expected in this architecture, is indeed beneficial for the enhancement of $\mathrm{H}_{2}$ production. Furthermore, the increase is the strongest at neutral conditions where the photobase effect is expected to manifest itself (cf. Fig. 4c). In the alkaline conditions the amount of evolved $\mathrm{H}_{2}$ is the same as for the components individually. At low $\mathrm{pH}$ the increase can also be observed but is much less pronounced. This is to be expected, as at high proton concentration, its transport is unlikely to be a limiting factor. The fact that the rate of $\mathrm{H}_{2}$ generation is highest at $\mathrm{pH} 3$ also indicates that proton transport could be limiting the process at its lower concentrations. In a control experiment, CD-acridine sample was illuminated through a 400-nm longpass filter which cuts off the acridine absorption and leaves only the tail of $\mathrm{CD}$ absorption. The very small observed $\mathrm{H}_{2}$ production confirms that the absorption by acridine is needed for an improvement. Two further control experiments were performed to check whether just the adsorption of an acridine-like compound has an effect. To this end, we have added two structurally similar molecules to CDs which, however, do not exhibit photobasic behavior at $\mathrm{pH}$ 7: anthracene and 9-aminoacridine. The former is not a photobase at all, the latter has $\mathrm{pK}_{\mathrm{a}}=10$ and even higher $\mathrm{pK}_{\mathrm{a}}^{*}$, so it cannot be protonated at $\mathrm{pH} 7^{50}$. In neither of these cases any increase in the $\mathrm{H}_{2}$ generation rate is observed (see Fig. 4d). This confirms that the photobasic property is critical to the enhancement. Finally, it is also important to establish the source of the evolved hydrogen. To this end, we performed a photocatalytic experiment in $\mathrm{D}_{2} \mathrm{O}$ instead of $\mathrm{H}_{2} \mathrm{O}$. We noted a decrease in hydrogen generation rate by a factor of 1.43 (cf. Supplementary Fig. 16). This result implies a strong kinetic isotope effect and is consistent with water, rather than CDs or acridine, as the source of protons for the produced hydrogen ${ }^{51}$.

\section{Discussion}

The rate of proton transfer determined by the time-resolved absorption measurements is consistent with the literature results obtained for smaller N-heterocyclic photobases (e.g. quinoline) $)^{7,9}$ and for acridine derivatives (e.g. acridine orange) ${ }^{39}$. Several studies 
a
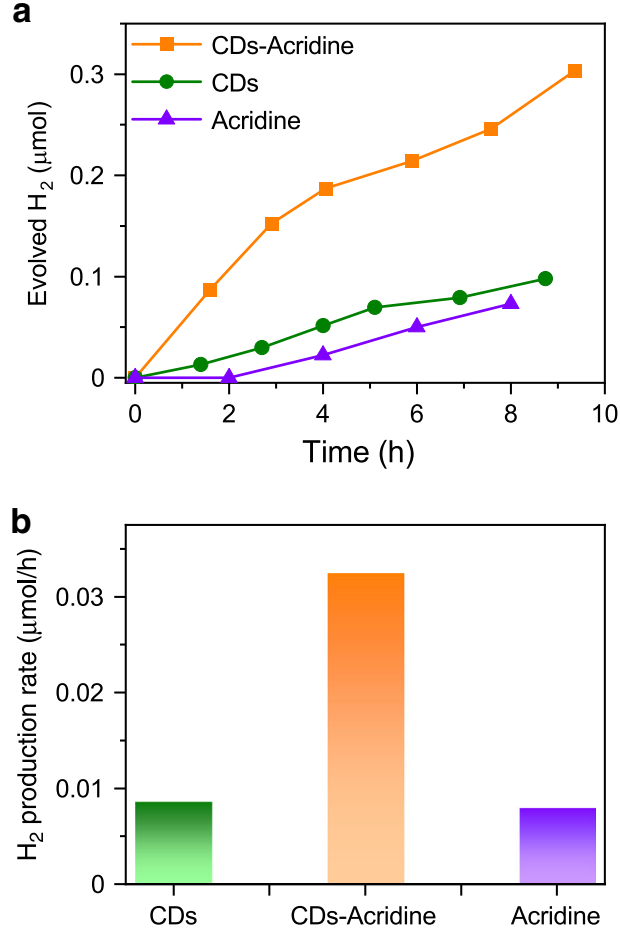

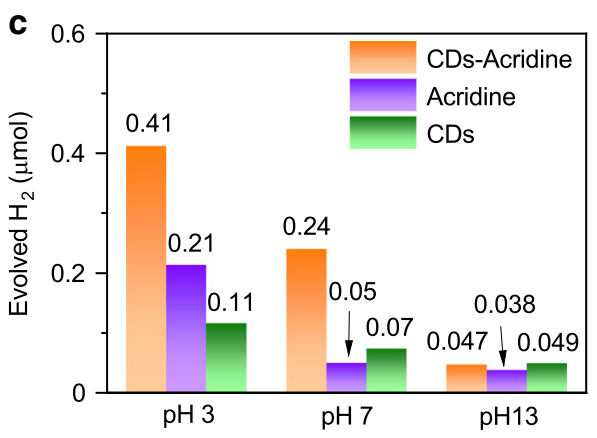

d

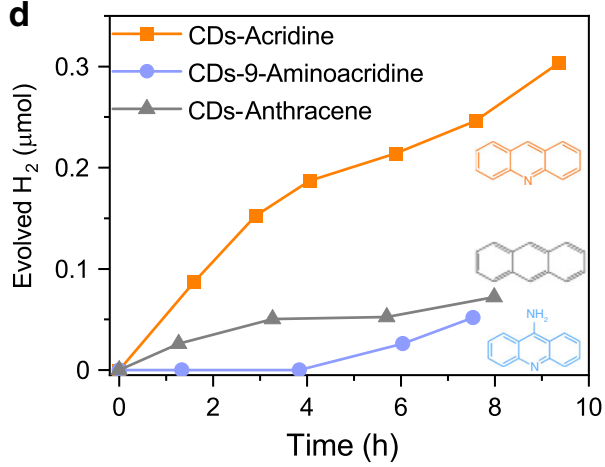

Fig. 4 Photocatalytic hydrogen generation. a Hydrogen evolution and $\mathbf{b}$ the average hydrogen production rate for CDs, acridine, and the mixture under $X \mathrm{e}$ lamp irradiation at $\mathrm{pH} 7$; $\mathbf{c}$ the total amount of evolved hydrogen over $6 \mathrm{~h}$ of illumination. $\mathbf{d}$ Comparison of hydrogen generation of mixtures of $\mathrm{CDs}$ with acridine, a non-photobase (anthracene) and a photobase (9-aminoacridine) with $\mathrm{pK}_{\mathrm{a}} 10$.

on photobase - proton donor (water and alcohols) systems also report that the proton transfer can be accompanied or quickly followed by electron transfer from the same molecule $39,52,53$. The concurrent electron transfer might also explain why the protonation rate is faster than reported earlier for acridine ${ }^{42,54}$. It may facilitate a charge transfer complex pathway which is usually involved in picoscond timescale protonation processes ${ }^{52,55}$. Eventually, the resulting radical (e.g. Acr- $\mathrm{H}^{\bullet}$ ) can undergo lightdriven dissociation and lead to molecular hydrogen via Heyrovsky mechanism $^{56,57}$. Acridine can only attract one proton, but in larger polycyclic structures with multiple photobasic moieties potentially in vicinity, Tafel reaction to form $\mathrm{H}_{2}$ may also be possible ${ }^{58}$. Here, in the $\mathrm{CD}$-acridine system, there is second feasible source of the electron, i.e., the photoexcited CDs. The cyclic voltammetry measurements provide evidence that the band alignment is appropriate for the electron transfer from CD to acridine. It is anticipated that the electron transfer would be even more efficient for a photobasic moiety integrated (or embedded) in the aromatic domains of the CDs, that was postulated to occur in $\mathrm{N}$-doped $\mathrm{CDs}^{23}$, and concurred by our experiments on the efficiency of the CDs with embedded acridine. The system comprising a $\mathrm{CD}$ and a photobase can thus transfer the two participating particles, a proton and an electron, to the reaction site at approximately the same time from two different sources, as depicted in Fig. 1. Under such scenario, any of the components would not have to wait for another, as they are delivered when needed, upon photoexcitation. This reduces the chances of parasitic charge recombination that inhibits photocatalytic activity. The second advantage of the CD-photobase system over a direct electron transfer from the proton donor is a generation of oxidized form of the latter (e.g. hydroxyl or alcoxyl radicals) in the vicinity of the reduction site. These reactive species could induce a back reaction with the $\mathrm{H}$ atom, reversing the benefit of the process. In a combined CD-photobase system, the photogenerated hole in the $\mathrm{CD}$ can be taken away by the scavenger at a more distant site, elsewhere on the dot. In such circumstances, the back reaction would be less likely. Moreover, it is worth noting that, while the experiments in the paper have focused on $\mathrm{H}_{2}$ generation, the proton transfer is an integral part also of the $\mathrm{CO}_{2}$ photocatalytic reduction. Thus, it can be surmised that the ability to induce the proton transfer via an increased basicity in the excited state could also be exploited in this process for increasing efficiency or selectivity of the $\mathrm{CO}_{2}$ reduction pathways.

In summary, we have shown that the proton transport in photocatalytic systems can be controlled by light. In the excited state photobase molecules can abstract a proton from the water within tens of picoseconds. This enables very fast - concerted or in quick succession - proton-coupled electron transfer and thereby substantially increases the rate of the overall photocatalytic process. We demonstrate this effect by fabricating nitrogen-free CDs and then including a model photobase, acridine, in the photocatalyst. This increases $4-10$-fold the $\mathrm{H}_{2}$ generation rate of the sample in the neutral conditions. As photobasic moieties are building blocks of many photocatalytically active nitrogen-containing CDs and of carbon nitrides, we propose that the photobasic effect may be an important part of the mechanism of the process on these materials and may partially account for their success in the photocatalytic $\mathrm{H}_{2}$ production. We believe that the uncovering of this mechanism could contribute to an improved design of the carbon nanomaterial-based photocatalysts and lead to further enhancements in activity.

\section{Methods}

Materials. Polyethylene glycol (PEG, average Mn 400 and 600, ACS reagent $\geq 99.5 \%$ ), ethanol, methanol, acridine, anthracene, 9-aminoacridine, and deuterium oxide $(99.9 \%$ atom D) were purchased from Sigma Aldrich and used without 
further purification. Distilled water (ultrapure) was used for the synthesis and for diluting samples for characterization. Methanol was used as a hole scavenger in the photocatalysis experiments.

Synthesis of CDs. The nitrogen-free CDs were synthesized by a straightforward pyrolysis method. Briefly, $318 \mathrm{mg}$ of waxy solid PEG600 was dissolved in $10 \mathrm{~mL}$ ethanol. Then the transparent solution was transferred into a $15-\mathrm{mL}$ poly(paraphenol)-lined stainless-steel autoclave and heated to $200^{\circ} \mathrm{C}$ for $2 \mathrm{~h}$. Afterwards, it was cooled down naturally to room temperature. The obtained solution was purified by centrifugation at $5000 \mathrm{rpm}$ for $5 \mathrm{~min}$ with only the supernatant taken for further characterization. To correlate the optical properties of CDs with photocatalytic activity in water, ethanol was removed by evaporation in $\mathrm{N}_{2}$ flow and the sample was re-dispersed in the same amount of distilled water.

CDs-Acridine samples were prepared by two methods. In the first method, the aqueous solution of CDs was mixed with the saturated acridine solution in water in $1: 3(\mathrm{v} / \mathrm{v})$ ratio, and then stirred with a magnetic bar to obtain a homogeneous solution. In the second method, acridine (same amount as in the first method) was mixed with PEG 400 in ethanol. This mixture was subsequently used as a reactant instead if pure PEG in the procedure that was the same as in CDs synthesis, described above. Ethanol in the sample was also removed and replaced with same amount of distilled water.

Morphological and optical characterizations. For electron microscopy measurements, the samples were first centrifuged at $5000 \operatorname{rot~min}^{-1}$ for $5 \mathrm{~min}$. Then an aliquot of the supernatant was drop-cast on a carbon coated copper grid and allowed to dry in the ambient environment. The transmission electron microscopy (TEM) and high-resolution transmission electron microscopic (HR-TEM) images were taken by a Titan Themis at $120 \mathrm{kV}$ accelerating voltage. UV-vis absorption measurements were carried out with a Varian Cary 5000 UV-Vis-IR spectrometer. A Horiba Jobin Yvon Fluorolog-3 FL3-22 spectrometer with a 450W Xe lamp was used to acquire the photoluminescence spectra. Its double monochromators for both excitation and emission were mounted at $90^{\circ}$ angle with a water-cooled photomultiplier tube. The resulting spectra were calibrated with excitation intensities and corrected. To perform time-resolved fluorescence measurements, a home-made device with a white light laser (NKT SuperK Extreme EXR-20), extended with an EXTEND-UV box, was used. The diluted samples were excited with $350 \mathrm{~nm}$ laser with a repetition rate of $5.56 \mathrm{MHz}$. The signal was detected with a Princeton Instruments monochromator fiber connected to a low-noise Avalanche Photodiode from Excelitas.

Femtosecond transient absorption spectroscopy. Ultrafast transient absorption measurements in the present investigation has been conducted in a custom-built transient absorption setup from Newport. A multi-pass Ti: Sapphire femtosecond regenerative amplifier laser system (Libra $\mathrm{HE}+$ from Coherent Inc.) produces $\sim 100$ fs laser pulse having $800-\mathrm{nm}$ central wavelength and at $1 \mathrm{kHz}$ repetition rate. A fraction of the 800-nm beam was passed through a spectrally tunable optical parametric amplifier (OPA) to produce the pump pulses at $350 \mathrm{~nm}$. The near UVvisible probe pulses (300-750 $\mathrm{nm}$ ) were generated by focusing another part of the initial 800-nm beam through a 2-mm-thick motorized $\mathrm{CaF}_{2}$ crystal. Further, the near UV-visible beam is split to two beams, probe and reference, to achieve better signal-to-noise ratios. The pump pulses were overlapped spatially and temporally on the sample with the probe pulses after passing through a $0.5-\mathrm{kHz}$ optical chopper to read out the changes in probe transmission for each pump-probe cycle. To provide a time delay between the two pulses, a motorized optical delay line was positioned in the probe arm. MS260i spectrograph by Newport, Inc. acquired the transmitted signal used for further analysis of the data. Finally, the changes in the absorptions $(\Delta \mathrm{A})$ of the excited state were computed by subtracting the absorptions of excited and unexcited samples.

Photocatalytic hydrogen generation. The experiments were conducted in a custom-built metal-free quartz cuvette. The $4.4 \mathrm{ml}$ cuvette was filled with $2 \mathrm{ml}$ aqueous solution containing $10 \%(\mathrm{v} / \mathrm{v})$ methanol and placed $35 \mathrm{~cm}$ from the illumination source. A 450W Xe lamp was used for broad-band UV-Vis illumination without any filter, but as shown in the emission spectrum (Supplementary Fig. 17), the intensity decreases significantly for wavelengths shorter than $350 \mathrm{~nm}(10-15$ $\mathrm{mW}$ intensity measured with $320 \mathrm{~nm}$ fluorescence filter). Prior to illumination, the cuvette was purged with argon for $1 \mathrm{~min}$ to remove air completely. The calculated illumination area was $1 \mathrm{~cm}^{2}$. The generated hydrogen was detected with a Shimadzu GC 2014 gas chromatograph by taking $10 \mu \mathrm{L}$ aliquots of the headspace from the cuvette at regular time intervals and injecting into the GC. Argon was used as a carrier gas in the chromatograph.

\section{Data availability}

Data is available from the corresponding author upon reasonable request.

Received: 1 November 2019; Accepted: 17 August 2020;

Published online: 14 October 2020

\section{References}

1. Chen, X., Shen, S., Guo, L. \& Mao, S. S. Semiconductor-based photocatalytic hydrogen generation. Chem. Rev. 110, 6503-6570 (2010).

2. Stolarczyk, J. K., Bhattacharyya, S., Polavarapu, L. \& Feldmann, J. Challenges and prospects in solar water splitting and $\mathrm{CO}_{2}$ reduction with inorganic and hybrid nanostructures. ACS Catal. 8, 3602-3635 (2018).

3. Ozin, G. A. Throwing new light on the reduction of $\mathrm{CO}_{2}$. Adv. Mater. 27, 1957-1963 (2015)

4. Aresta, M., Dibenedetto, A. \& Angelini, A. Catalysis for the valorization of exhaust carbon: from $\mathrm{CO}_{2}$ to chemicals, materials, and fuels. technological use of $\mathrm{CO}_{2}$. Chem. Rev. 114, 1709-1742 (2014).

5. Cook, T. R. et al. Solar energy supply and storage for the legacy and nonlegacy worlds. Chem. Rev. 110, 6474-6502 (2010).

6. Pannwitz, A. \& Wenger, O. S. Proton-coupled multi-electron transfer and its relevance for artificial photosynthesis and photoredox catalysis. Chem. Commun. 55, 4004-4014 (2019).

7. Hunt, J. R. \& Dawlaty, J. M. Photodriven deprotonation of alcohols by a quinoline photobase. J. Phys. Chem. A 122, 7931-7940 (2018).

8. Hunt, J. R., Tseng, C. \& Dawlaty, J. M. Donor-acceptor preassociation, excited state solvation threshold, and optical energy cost as challenges in chemical applications of photobases. Faraday Discuss. 216, 252-268 (2019)

9. Driscoll, E. W., Hunt, J. R. \& Dawlaty, J. M. Proton capture dynamics in quinoline photobases: substituent effect and involvement of triplet states. J. Phys. Chem. A 121, 7099-7107 (2017)

10. Lennox, J. C., Kurtz, D. A., Huang, T. \& Dempsey, J. L. Excited-state protoncoupled electron transfer: different avenues for promoting proton/electron movement with solar photons. ACS Energy Lett. 2, 1246-1256 (2017).

11. Costi, R., Saunders, A. E. \& Banin, U. Colloidal hybrid nanostructures: a new type of functional materials. Angew. Chem. Int. Ed. 49, 4878-4897 (2010).

12. Osterloh, F. E. Inorganic nanostructures for photoelectrochemical and photocatalytic water splitting. Chem. Soc. Rev. 42, 2294-2320 (2013)

13. Wilker, M. B., Schnitzenbaumer, K. J. \& Dukovic, G. Recent progress in photocatalysis mediated by colloidal II-VI nanocrystals. Isr. J. Chem. 52 1002-1015 (2012).

14. Fernando, K. A. S. et al. Carbon quantum dots and applications in photocatalytic energy conversion. ACS Appl. Mater. Interfaces 7, 8363-8376 (2015).

15. Arcudi, F., Đorđević, L. \& Prato, M. Design, synthesis, and functionalization strategies of tailored carbon nanodots. Acc. Chem. Res. 52, 2070-2079 (2019)

16. Cao, L. et al. Carbon dots for energy conversion applications. J. Appl. Phys. 125, 220903 (2019)

17. Cadranel, A., Margraf, J. T., Strauss, V., Clark, T. \& Guldi, D. M. Carbon nanodots for charge-transfer processes. Acc. Chem. Res. 52, 955-963 (2019).

18. LeCroy, G. E. et al. Functionalized carbon nanoparticles: syntheses and applications in optical bioimaging and energy conversion. Coord. Chem. Rev. 320-321, 66-81 (2016).

19. Lim, S. Y., Shen, W. \& Gao, Z. Carbon quantum dots and their applications. Chem. Soc. Rev. 44, 362-381 (2015).

20. Fu, M. et al. Carbon dots: a unique fluorescent cocktail of polycyclic aromatic hydrocarbons. Nano Lett. 15, 6030-6035 (2015).

21. Schneider, J. et al. Molecular fluorescence in citric acid-based carbon dots. $J$ Phys. Chem. C 121, 2014-2022 (2017)

22. Ehrat, F. et al. Tracking the source of carbon dot photoluminescence: aromatic domains versus molecular fluorophores. Nano Lett. 17, 7710-7716 (2017).

23. Bhattacharyya, S. et al. Effect of nitrogen atom positioning on the trade-off between emissive and photocatalytic properties of carbon dots. Nat. Commun 8, 1401 (2017).

24. Rabe, E. J., Corp, K. L., Sobolewski, A. L., Domcke, W. \& Schlenker, C. W. Proton-coupled electron transfer from water to a model heptazine-based molecular photocatalyst. J. Phys. Chem. Lett. 9, 6257-6261 (2018).

25. Wang, Y. et al. Current understanding and challenges of solar-driven hydrogen generation using polymeric photocatalysts. Nat. Energy 4, 746-760 (2019).

26. Ehrmaier, J., Karsili, T. N. V., Sobolewski, A. L. \& Domcke, W. Mechanism of photocatalytic water splitting with graphitic carbon nitride: photochemistry of the heptazine-water complex. J. Phys. Chem. A 121, 4754-4764 (2017).

27. Schlomberg, H. et al. Structural insights into poly(heptazine imides): a lightstoring carbon nitride material for dark photocatalysis. Chem. Mater. 31, 7478-7486 (2019)

28. Lau, V. W.-h et al. Rational design of carbon nitride photocatalysts by identification of cyanamide defects as catalytically relevant sites. Nat Commun. 7, 12165 (2016).

29. Lau, V. W.-h et al. Dark photocatalysis: storage of solar energy in carbon nitride for time-delayed hydrogen generation. Angew. Chem. Int. Ed. 56, 510-514 (2017).

30. Dvoranová, D. et al. EPR investigations of polymeric and $\mathrm{H}_{2} \mathrm{O}_{2}$-modified $\mathrm{C}_{3} \mathrm{~N}_{4}$-based photocatalysts. J. Photochem. Photobiol. A 375, 100-113 (2019). 
31. Xu, J., Zhang, L., Shi, R. \& Zhu, Y. Chemical exfoliation of graphitic carbon nitride for efficient heterogeneous photocatalysis. J. Mater. Chem. A 1, 14766-14772 (2013).

32. Zhang, Y., Thomas, A., Antonietti, M. \& Wang, X. Activation of carbon nitride solids by protonation: morphology changes, enhanced ionic conductivity, and photoconduction experiments. J. Am. Chem. Soc. 131, 50-51 (2009).

33. Ong, W.-J. et al. Unravelling charge carrier dynamics in protonated $\mathrm{g}^{-} \mathrm{C}_{3} \mathrm{~N}_{4}$ interfaced with carbon nanodots as co-catalysts toward enhanced photocatalytic $\mathrm{CO} 2$ reduction: a combined experimental and first-principles DFT study. Nano Res. 10, 1673-1696 (2017).

34. Perrin, D. D. Dissociation Constants Of Organic Bases In Aqueous Solution. (Butterworths, London, 1965).

35. Artyukhov, V. Y. et al. A combined theoretical and experimental study on molecular photonics. Russ. Phys. J. 51, 1097-1111 (2008).

36. Rubio-Pons, Ò., Serrano-Andrés, L. \& Merchán, M. A theoretical insight into the photophysics of acridine. J. Phys. Chem. A 105, 9664-9673 (2001).

37. Liu, X., Karsili, T. N. V., Sobolewski, A. L. \& Domcke, W. Photocatalytic water splitting with the acridine chromophore: a computational study. J. Phys. Chem. B 119, 10664-10672 (2015).

38. Liu, X., Karsili, T. N. V., Sobolewski, A. L. \& Domcke, W. Photocatalytic water splitting with acridine dyes: Guidelines from computational chemistry. Chem. Phys. 464, 78-85 (2016).

39. Eisenhart, T. T. \& Dempsey, J. L. Photo-induced proton-coupled electron transfer reactions of acridine orange: comprehensive spectral and kinetics analysis. J. Am. Chem. Soc. 136, 12221-12224 (2014).

40. Fan, R.-J., Sun, Q., Zhang, L., Zhang, Y. \& Lu, A.-H. Photoluminescent carbon dots directly derived from polyethylene glycol and their application for cellular imaging. Carbon 71, 87-93 (2014).

41. Baker, S. N. \& Baker, G. A. Luminescent carbon nanodots: emergent nanolights. Angew. Chem. Int. Ed. 49, 6726-6744 (2010).

42. Pines, E., Huppert, D., Gutman, M., Nachliel, N. \& Fishman, M. The pOH jump: determination of deprotonation rates of water by 6-methoxyquinoline and acridine. J. Phys. Chem. 90, 6366-6370 (1986).

43. Yang, L. et al. One pot synthesis of highly luminescent polyethylene glycol anchored carbon dots functionalized with a nuclear localization signal peptide for cell nucleus imaging. Nanoscale 7, 6104-6113 (2015).

44. Kasama, K. et al. Relaxation mechanism of excited acridine in nonreactive solvents. J. Phys. Chem. 85, 1291-1296 (1981).

45. Ryan, E. T., Xiang, T., Johnston, K. P. \& Fox, M. A. Absorption and fluorescence studies of acridine in subcritical and supercritical water. J. Phys. Chem. A 101, 1827-1835 (1997).

46. Rak, J., Blazejowski, J. \& Zauhar, R. J. Theoretical studies on the prototropic tautomerism, structure, and features of acridine and 9-acridinamine free bases and their protonated forms. J. Org. Chem. 57, 3720-3725 (1992).

47. Hirata, Y. \& Tanaka, I. Build-up of T-T absorption of acridine following singlet excitation with a picosecond pulse. Chem. Phys. Lett. 41, 336-338 (1976).

48. Simon, T., Carlson, M. T., Stolarczyk, J. K. \& Feldmann, J. Electron transfer rate vs recombination losses in photocatalytic $\mathrm{H}_{2}$ generation on Pt-decorated CdS nanorods. ACS Energy Lett. 1, 1137-1142 (2016).

49. $\mathrm{Wu}, \mathrm{K}$. et al. Hole removal rate limits photodriven $\mathrm{H}_{2}$ generation efficiency in CdS-Pt and CdSe/CdS-Pt semiconductor nanorod-metal tip heterostructures. J. Am. Chem. Soc. 136, 7708-7716 (2014).

50. Casadio, R. \& Melandri, B. A. The behavior of 9-aminoacridine as an indicator of transmembrane $\mathrm{pH}$ difference in liposomes of natural bacterial phospholipids. J. Bioenerg. Biomembr. 9, 17-29 (1977).

51. Hisatomi, T., Takanabe, K. \& Domen, K. Photocatalytic water-splitting reaction from catalytic and kinetic perspectives. Catal. Lett. 145, 95-108 (2015).

52. Hammes-Schiffer, S. \& Stuchebrukhov, A. A. Theory of coupled electron and proton transfer reactions. Chem. Rev. 110, 6939-6960 (2010).

53. Rahman, M. Z. \& Mullins, C. B. Understanding charge transport in carbon nitride for enhanced photocatalytic solar fuel production. Acc. Chem. Res. 52, 248-257 (2019).

54. Munitz, N., Avital, Y., Pines, D., Nibbering, E. T. J. \& Pines, E. Cationenhanced deprotonation of water by a strong photobase. Isr. J. Chem. 49, 261-272 (2009).
55. Mohammed, O. F., Pines, D., Nibbering, E. T. J. \& Pines, E. Base-induced solvent switches in acid-base reactions. Angew. Chem. Int. Ed. 46, 1458-1461 (2007).

56. de Chialvo, M. R. G. \& Chialvo, A. C. Hydrogen evolution reaction: Analysis of the Volmer-Heyrovsky-Tafel mechanism with a generalized adsorption model. J. Electroanal. Chem. 372, 209-223 (1994).

57. Kou, T. et al. Carbon doping switching on the hydrogen adsorption activity of $\mathrm{NiO}$ for hydrogen evolution reaction. Nat. Commun. 11, 590 (2020).

58. Machado, S. A. S. \& Avaca, L. A. The hydrogen evolution reaction on nickel surfaces stabilized by H-absorption. Electrochim. Acta 39, 1385-1391 (1994).

\section{Acknowledgements}

This work was supported by the Bavarian State Ministry of Science, Research, and Arts through the grant "Solar Technologies go Hybrid (SolTech)", the EU ERANETMED program (project Hydrosol, Grant No. ENERG-11-132), the China Scholarship Counci (J. Fang), and the Alexander von Humboldt-Stiftung (T.D. and S.B.). We thank local research clusters and centers such as the Center of Nanoscience (CeNS) and e-conversion for providing communicative networking structures.

\section{Author contributions}

M.D. performed the HR-TEM measurements; J.Fang performed all other experiments, in part together with T.D. and S.B. for the time-resolved spectroscopic experiments and sample preparation, respectively. J.K.S. wrote the manuscript with the inputs and com ments from all other authors. J.K.S. and J.Feldmann supervised the work. All authors contributed to the design of the experiments, the interpretation of the results, and a discussion of the outline of the manuscript.

\section{Funding}

Open Access funding provided by Projekt DEAL.

\section{Competing interests}

The authors declare no competing interests.

\section{Additional information}

Supplementary information is available for this paper at https://doi.org/10.1038/s41467020-18583-6.

Correspondence and requests for materials should be addressed to J.K.S.

Peer review information Nature Communications thanks Pradip Pachfule and other, anonymous, reviewer(s) for their contributions to the peer review of this work.

Reprints and permission information is available at http://www.nature.com/reprints

Publisher's note Springer Nature remains neutral with regard to jurisdictional claims in published maps and institutional affiliations.

(c) (i) Open Access This article is licensed under a Creative Commons Attribution 4.0 International License, which permits use, sharing, adaptation, distribution and reproduction in any medium or format, as long as you give appropriate credit to the original author(s) and the source, provide a link to the Creative Commons license, and indicate if changes were made. The images or other third party material in this article are included in the article's Creative Commons license, unless indicated otherwise in a credit line to the material. If material is not included in the article's Creative Commons license and your intended use is not permitted by statutory regulation or exceeds the permitted use, you will need to obtain permission directly from the copyright holder. To view a copy of this license, visit http://creativecommons.org/ licenses/by/4.0/.

(c) The Author(s) 2020 